\title{
Facteurs de transfert du césium au lait et à la viande déterminés à partir de mesures faites en France en 2011 à la suite de l'accident de Fukushima-Daichi
}

\author{
L. SAEY ${ }^{1}$, L. POURCELOT ${ }^{1}$, V. PARACHE ${ }^{1}$, S. ROUSSEL-DEBET ${ }^{1}$, \\ R. GURRIARAN ${ }^{2}$, D. ORJOLLET ${ }^{3}$, F. LEBLANC ${ }^{4}$, PH. RENAUD $^{1}$
}

(Manuscrit reçu le 20 novembre 2012, accepté le 4 février 2013)

RÉSUMÉ Afin d'évaluer les conséquences des rejets radioactifs provenant des réacteurs acciAfin d'évaluer les conséquences des rejets radioactifs provenant des réacteurs acci-
dentés de la centrale nucléaire de Fukushima-Daichi sur l'environnement terrestre francais, l'IRSN a renforcé son plan de surveillance en échantillonnant notamment de la végétation de prairie, du lait et de la viande des troupeaux en pâture entre avril et novembre 2011. Ces prélèvements et ces mesures ont, entre autres, permis d'évaluer dans des conditions de dépôts réels la chronologie des transferts du césium-134 de l'herbe au lait et à la viande. Les premières mesures significatives en ${ }^{134} \mathrm{Cs}$ apparaissent dans l'herbe et dans le lait cinq à sept jours après la première mesure significative dans l'air. Les activités maximales atteignent $0,4 \mathrm{~Bq} \cdot \mathrm{kg}^{-1} \mathrm{~d}$ 'herbe fraiche et $0,028 \mathrm{~Bq} \cdot \mathrm{L}^{-1}$ dans le lait. Dans la viande, les premières mesures significatives apparaissent mi-avril avec une activité maximale mesurée de $0,036 \mathrm{~Bq} \cdot \mathrm{kg}^{-1}$, avec de fortes variations d'une espèce animale à une autre. Les coefficients de transfert moyens calculés sont proches de ceux disponibles dans la littérature avec $0,22 \mathrm{j} \cdot \mathrm{kg}^{-1}$ pour la viande de mouton, et de 0,09 et $0,0014 \mathrm{j} . \mathrm{L}^{-1}$ pour le lait de chèvre et de vache, respectivement.

ABSTRACT Transfer factors of cesium in milk and meat determined from measurements made in France in 2011 following the Fukushima-Daichi accident.

In order to evaluate the consequences in the terrestrial environment in France after the releases from the damaged reactors of Fukushima-Daichi, the IRSN carried out a monitoring program involving the sampling of grassland vegetation, and milk and meat from outdoor grazing animals, between April and November 2011. This field data allowed the evaluation of the timing of the transfer and the accumulation of ${ }^{134} \mathrm{Cs}$ over time in milk and meat in the real conditions of the radioactive deposition. The first ${ }^{134} \mathrm{Cs}$ detections were observed in vegetation and milk five to seven days after the detection in the air. The activities reach $0.4 \mathrm{~Bq} \cdot \mathrm{kg}^{-1}$ and $0.028 \mathrm{~Bq} \cdot \mathrm{L}^{-1}$ in grass and milk, respectively. In meat, the first detections were observed by mid-April. The activities reach $0.036 \mathrm{~Bq} . \mathrm{kg}^{-1}$, with large variations according to the species. The mean calculated transfer coefficients are close to those available in the literature, with $0.22 \mathrm{~d}_{\mathrm{kg}} \mathrm{g}^{-1}$ for mutton and 0.09 and $0.0014 \mathrm{~d} . \mathrm{L}^{-1}$ for goat's and cow's milk, respectively.

Keywords: Fukushima / cesium / milk / meat / France

\footnotetext{
IRSN, Laboratoire d'Études Radioécologiques du milieu Continental et Marin, Bât. 153, CEN Cadarache, BP 3, 13115 St Paul les Durance, France.

IRSN, Laboratoire de Mesure de la Radioactivité dans l'Environnement, Bât. 501, Bois des Rames, 91400 Orsay, France. IRSN, Laboratoire de Biogéochimie, Biodisponibilité et Transferts des radionucléides, Bât. 153, CEN Cadarache, BP 3, 13115 St Paul les Durance, France.

4 IRSN, Groupe d'intervention en radioprotection opérationnelle - SUD-OUEST, Site du Centre Hospitalier, Route de Villeneuve sur Lot, BP 27, 47002 Agen, France.
} 


\section{Introduction}

Au printemps 2011, l'IRSN a réalisé, dans le cadre d'un plan de surveillance renforcé (IRSN, 2011), des prélèvements et des mesures de différents compartiments de l'environnement terrestre français pour évaluer les conséquences des rejets radioactifs provenant des réacteurs accidentés de la centrale nucléaire de Fukushima-Daichi. Les mesures in situ acquises à cette période ont permis d'évaluer le passage des masses d'air contaminées sur le territoire métropolitain (Masson et al., 2011) et le transfert de l'iode-131 dans les plantes et le lait (Parache et al., 2011). La présente publication est axée principalement sur les résultats des mesures de l'activité en césium-134 dans la végétation de prairie, le lait et la viande des troupeaux.

En effet, des deux principaux isotopes du césium mesurables en spectrométrie gamma $\left({ }^{137} \mathrm{Cs}\right.$ et $\left.{ }^{134} \mathrm{Cs}\right)$, le ${ }^{134} \mathrm{Cs}$ s'avère être, en France, l'unique traceur du césium rejeté par les réacteurs de Fukushima-Daichi qui, du fait de sa courte période radioactive $(2,1$ ans) avait disparu par décroissance après avoir été émis avec les retombées de l'accident de Tchernobyl. A contrario, le césium-137, provenant des dépôts liés à ces retombées ainsi qu'à celles plus anciennes des essais atmosphériques des années 60 et dont la période radioactive est plus longue (30 ans), est encore mesuré en routine dans l'environnement.

L'intérêt de ces mesures dans la végétation, le lait et la viande, réalisées dans les conditions d'un dépôt radioactif réel est (1) de comparer l'évolution des activités dans ces différents compartiments au cours du temps et (2) d'évaluer l'intensité des transferts du césium entre ces compartiments, c'est-à-dire de calculer les coefficients de transfert dans le lait et la viande.

\section{Méthodes de prélèvements et de mesure}

Les prélèvements de végétation de prairie (58 échantillons), de lait (40 échantillons) et de viande de troupeaux en pâture (19 échantillons) ont été réalisés entre le 4 avril et le 7 novembre 2011, c'est-à-dire avant, pendant et après le passage des masses d'air contaminées provenant du Japon.

Trois à cinq kilogrammes de viande fraiche d'espèces bovines et ovines ont été prélevés périodiquement (mensuellement dans la mesure du possible) sur différents sites : du veau de lait dans la région de Saint-Alban (Isère), du taureau de Camargue vers Saint-Rémy-de-Provence (Bouches-du-Rhône), du bœuf (Blonde d'Aquitaine) dans la région d'Agen (Lot-et-Garonne) et du mouton (brebis) à proximité du Centre d'Etude Nucléaire de Cadarache (Bouches-duRhône) (Fig. 1). Les troupeaux ont été sélectionnés en s'assurant au préalable 


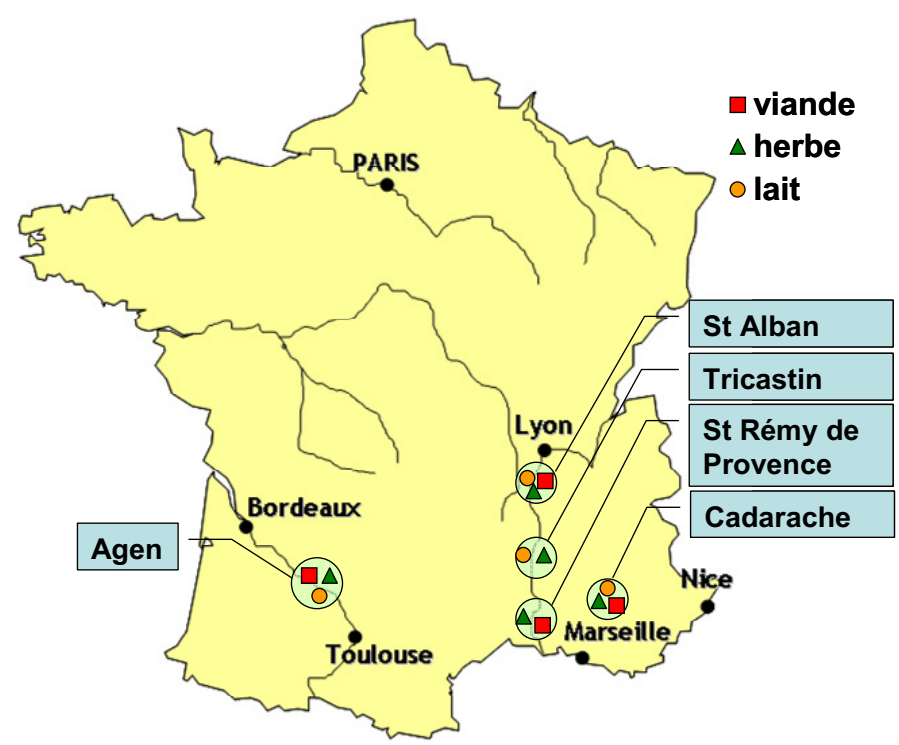

Figure 1 - Localisation des prélèvements de lait, de viande et d'herbe dans le sud de la France.

Location of milk, meat and grass sampling sites in the south of France.

que l'affourragement provenait directement des prairies sur lesquelles avait été échantillonnée l'herbe. Compte-tenu des conditions climatiques lors de la période d'investigation (débutant en mars), seules les stations de prélèvement situées au sud de la France répondaient à ce critère. Pour chaque station, les échantillons de viande proviennent de bétail de même espèce, d'âge et de corpulences similaires pour éviter une trop grande variabilité dans les résultats de mesure liée à l'âge des sujets.

Trois litres par échantillon de lait de vache ou de chèvre ont été prélevés à la même période sur les sites d'Agen et à proximité des sites nucléaires de Tricastin (Drôme), de Cadarache et de Saint-Alban. Sur l'ensemble des sites, des prélèvements d'herbe (surface de $1 \mathrm{~m}^{2} \mathrm{à} 3 \mathrm{~m}^{2}$ ) ont été effectués dans les parcelles broutées par les troupeaux.

Les échantillons de viande et d'herbe ont été séchés à $80^{\circ} \mathrm{C}$ puis incinérés à $480^{\circ} \mathrm{C}$. Les échantillons de lait ont été lyophilisés avant incinération. Les cendres obtenues ont été conditionnées dans des géométries de comptage de 30 ou $60 \mathrm{~mL}$. L'activité en ${ }^{134} \mathrm{Cs}$ a été mesurée dans ces géométries à l'aide de détecteurs gamma germanium hyper pur à bas bruit de fond dont dispose le Laboratoire de Mesure de la Radioactivité dans l'Environnement (LMRE) de l'IRSN (Orsay). 
Les incertitudes de mesure sont déterminées par le LMRE de l'IRSN, selon la méthode préconisée par le groupe de travail 1 du comité commun pour les guides en métrologie (JCGM, 2008).

Les limites de détection sont déterminées selon les méthodes préconisées par la norme AFNOR NF ISO 11929 (AFNOR, 2010). Elles sont définies spécifiquement pour chaque mesure et varient en fonction des conditions de mesure et des spécificités de chaque échantillon.

\section{Résultats}

Les activités en ${ }^{134} \mathrm{Cs}$ mesurées dans les différentes matrices (herbe, lait et viande) provenant des cinq sites étudiés sont reportées dans le tableau I. Les variations des activités de ce radioélément en fonction du temps sont représentées sur la figure 2.

\subsection{Activité dans la végétation et le lait}

Les premières mesures significatives apparaissent entre le 28 mars et le $1^{\mathrm{er}}$ avril 2011, dans l'herbe et dans le lait soit cinq à sept jours après la première mesure significative dans l'air (Masson et al., 2011). Les activités maximales de $0,406 \mathrm{~Bq} \cdot \mathrm{kg}^{-1} \mathrm{~d}$ 'herbe fraiche et de $0,028 \mathrm{~Bq} . \mathrm{L}^{-1}$ dans le lait sont rapidement atteintes, quatre jours après le début des dépôts, selon les sites étudiés. L'activité dans ces matrices diminue au fil du temps et le ${ }^{134}$ Cs n'est plus mesurable (activité inférieure à la limite de détection) dans la plupart des échantillons d'herbe et de lait à partir de la mi-mai.

Parmi les sites étudiés, c'est à Agen qu'ont été mesurées les activités les plus élevées dans l'herbe des prairies $\left(0,057\right.$ à $\left.0,406 \mathrm{~Bq} \cdot \mathrm{kg}^{-1}\right)$ et le lait de vache $(0,005$ à $\left.0,028 \mathrm{~Bq} \cdot \mathrm{L}^{-1}\right)$ (Fig. 3). En comparaison, des activités un peu plus faibles sont observées sur les sites du sud-est de la France, comme à proximité des sites de Tricastin (herbe : 0,027 à $0,085 \mathrm{~Bq} \cdot \mathrm{kg}^{-1}$; lait de chèvre : 0,005 à $0,013 \mathrm{~Bq} . \mathrm{L}^{-1}$ ) et de Cadarache (herbe : 0,030 à $0,059 \mathrm{~Bq} \cdot \mathrm{kg}^{-1}$; lait de chèvre : 0,008 à $0,011 \mathrm{~Bq} \cdot \mathrm{L}^{-1}$ ).

\subsection{Activité dans la viande}

Dans la viande, les premières mesures significatives apparaissent plus tardivement que dans les autres matrices (mi-avril) (Fig. 2) en raison de l'accumulation progressive du césium dans les muscles. L'activité maximale mesurée dans la viande de mouton est de $0,037 \mathrm{~Bq} \cdot \mathrm{kg}^{-1}$ frais. Comme dans la végétation et le lait, l'activité en ${ }^{134} \mathrm{Cs}$ dans la viande diminue et, à partir de la mi-juillet, il n'est plus mesuré dans cette matrice. Les activités en ${ }^{134} \mathrm{Cs}$ observées dans la viande de mouton prélevée à proximité du site de Cadarache confirment cette évolution 
FACTEURS DE TRANSFERT DU CÉSIUM AU LAIT ET À LA VIANDE

TABLEAU I

Activité en ${ }^{134} \mathrm{Cs}$ des échantillons de viande, de lait et d'herbe prélevés dans le sud de la France en 2011.

${ }^{134} \mathrm{Cs}$ activity measured in samples of meat, milk and grass collected in the south of France in 2011.

\begin{tabular}{|c|c|c|c|c|c|}
\hline $\begin{array}{l}\text { Stations } \\
\text { Espèces }\end{array}$ & Date & Bq. $\mathrm{kg}^{-1}$ frais ou Bq. $\mathrm{L}^{-1}$ & $\begin{array}{l}\text { Stations } \\
\text { Espèces }\end{array}$ & Date & Bq.kg ${ }^{-1}$ frais ou Bq. $\mathrm{L}^{-1}$ \\
\hline \multicolumn{3}{|l|}{ Agen } & \multicolumn{3}{|l|}{ Tricastin } \\
\hline \multirow[t]{17}{*}{ Herbe } & $21 / 03$ & $<0,032$ & \multirow[t]{14}{*}{ Herbe } & $26 / 03$ & $<0,092$ \\
\hline & $25 / 03$ & $<0,031$ & & $29 / 03$ & $0,085 \pm 0,035$ \\
\hline & $28 / 03$ & $0,211 \pm 0,078$ & & $01 / 04$ & $0,026 \pm 0,009$ \\
\hline & $30 / 03$ & $0,378 \pm 0,065$ & & $04 / 04$ & $0,069 \pm 0,041$ \\
\hline & $01 / 04$ & $0,400 \pm 0,068$ & & $06 / 04$ & $0,034 \pm 0,01$ \\
\hline & $04 / 04$ & $0,230 \pm 0,060$ & & 08/04 & $0,027 \pm 0,013$ \\
\hline & $06 / 04$ & $0,406 \pm 0,150$ & & $11 / 04$ & $<0,076$ \\
\hline & $08 / 04$ & $0,283 \pm 0,060$ & & $14 / 04$ & $<0,100$ \\
\hline & $12 / 04$ & $0,204 \pm 0,031$ & & $19 / 04$ & $<0,050$ \\
\hline & $15 / 04$ & $0,301 \pm 0,037$ & & $28 / 04$ & $<0,026$ \\
\hline & $20 / 04$ & $0,145 \pm 0,067$ & & $03 / 05$ & $<0,071$ \\
\hline & $28 / 04$ & $0,135 \pm 0,078$ & & $10 / 05$ & $<0,033$ \\
\hline & $11 / 05$ & $0,057 \pm 0,028$ & & $07 / 07$ & $<0,080$ \\
\hline & $30 / 05$ & $<0,068$ & & $23 / 08$ & $<0,037$ \\
\hline & $21 / 07$ & $0,089 \pm 0,015$ & \multirow{15}{*}{$\begin{array}{l}\text { Lait de } \\
\text { chèvre }\end{array}$} & $26 / 03$ & $<0,011$ \\
\hline & $08 / 08$ & $<0,023$ & & $29 / 03$ & $<0,009$ \\
\hline & $09 / 11$ & $<0,023$ & & $01 / 04$ & $0,01 \pm 0,003$ \\
\hline \multirow{12}{*}{$\begin{array}{l}\text { Lait de } \\
\text { vache }\end{array}$} & $28 / 03$ & $<0,021$ & & $04 / 04$ & $0,01 \pm 0,003$ \\
\hline & $30 / 03$ & $0,020 \pm 0,006$ & & $06 / 04$ & $0,011 \pm 0,004$ \\
\hline & $01 / 04$ & $0,022 \pm 0,004$ & & $08 / 04$ & $0,013 \pm 0,004$ \\
\hline & $04 / 04$ & $0,027 \pm 0,006$ & & $11 / 04$ & $0,011 \pm 0,004$ \\
\hline & $06 / 04$ & $0,028 \pm 0,005$ & & $14 / 04$ & $<0,011$ \\
\hline & $08 / 04$ & $0,024 \pm 0,005$ & & $18 / 04$ & $0,013 \pm 0,004$ \\
\hline & $12 / 04$ & $0,019 \pm 0,006$ & & $28 / 04$ & $<0,033$ \\
\hline & $15 / 04$ & $0,012 \pm 0,004$ & & $03 / 05$ & $0,005 \pm 0,002$ \\
\hline & $20 / 04$ & $<0,010$ & & $10 / 05$ & $<0,015$ \\
\hline & $28 / 04$ & $<0,014$ & & $01 / 06$ & $<0,008$ \\
\hline & $11 / 05$ & $0,005 \pm 0,002$ & & 07/07 & $<0,009$ \\
\hline & $30 / 05$ & $<0,011$ & & $23 / 08$ & $<0.008$ \\
\hline \multirow{5}{*}{$\begin{array}{l}\text { Viande de } \\
\text { bœuf }\end{array}$} & $06 / 05$ & $<0,016$ & & & \\
\hline & $10 / 06$ & $<0,022$ & & & \\
\hline & $15 / 07$ & $0,007 \pm 0,003$ & & & \\
\hline & $03 / 08$ & $<0,012$ & & & \\
\hline & $04 / 11$ & $<0,012$ & & & \\
\hline
\end{tabular}


TABLEAU I

Suite.

Continued.

\begin{tabular}{|c|c|c|c|c|c|}
\hline $\begin{array}{l}\text { Stations } \\
\text { Espèces }\end{array}$ & Date & Bq. $\mathrm{kg}^{-1}$ frais ou Bq. $\mathrm{L}^{-1}$ & $\begin{array}{l}\text { Stations } \\
\text { Espèces }\end{array}$ & Date & $\mathrm{Bq} \cdot \mathrm{kg}^{-1}$ frais ou Bq.L.-1 \\
\hline \multicolumn{3}{|l|}{ Cadarache } & \multicolumn{3}{|c|}{ St Rémy de Provence } \\
\hline \multirow[t]{18}{*}{ Herbe } & $21 / 03$ & $<0,171$ & \multirow[t]{2}{*}{ Herbe } & $03 / 08$ & $<0,028$ \\
\hline & $25 / 03$ & $<0,025$ & & $14 / 09$ & $<0,050$ \\
\hline & $28 / 03$ & $0,041 \pm 0,011$ & \multirow{5}{*}{$\begin{array}{r}\text { Viande de } \\
\text { taureau }\end{array}$} & 04/04 & $<0,014$ \\
\hline & $30 / 03$ & $0,050 \pm 0,011$ & & $04 / 05$ & $0,009 \pm 0,005$ \\
\hline & $01 / 04$ & $0,059 \pm 0,012$ & & 09/06 & $<0,016$ \\
\hline & $04 / 04$ & $0,044 \pm 0,010$ & & $20 / 07$ & $<0,006$ \\
\hline & $06 / 04$ & $0,036 \pm 0,010$ & & 05/09 & $<0,014$ \\
\hline & 08/04 & $0,030 \pm 0,007$ & & & \\
\hline & $11 / 04$ & $0,037 \pm 0,012$ & \multirow{2}{*}{$\begin{array}{l}\text { Stations } \\
\text { Espèces }\end{array}$} & \multirow{2}{*}{ Date } & \multirow{2}{*}{ Bq.kg ${ }^{-1}$ frais ou Bq.L $L^{-1}$} \\
\hline & $13 / 04$ & $0,045 \pm 0,013$ & & & \\
\hline & $18 / 04$ & $0,032 \pm 0,013$ & \multicolumn{3}{|l|}{ St Alban } \\
\hline & $27 / 04$ & $<0,105$ & \multirow[t]{7}{*}{ Herbe } & $26 / 03$ & $<0,092$ \\
\hline & $01 / 06$ & $<0,027$ & & $29 / 03$ & $<0,096$ \\
\hline & 07/07 & $<0,048$ & & $01 / 04$ & $<0,190$ \\
\hline & $26 / 07$ & $<0,032$ & & $04 / 04$ & $0,217 \pm 0,074$ \\
\hline & $26 / 08$ & $<0,039$ & & $12 / 04$ & $<0,184$ \\
\hline & $06 / 10$ & $<0,061$ & & $19 / 04$ & $<0,192$ \\
\hline & $09 / 11$ & $<0,032$ & & $29 / 09$ & $<0,044$ \\
\hline \multirow{13}{*}{$\begin{array}{l}\text { Lait de } \\
\text { chèvre }\end{array}$} & $28 / 03$ & $<0,014$ & \multirow{3}{*}{$\begin{array}{r}\text { Viande de } \\
\text { veau }\end{array}$} & $18 / 04$ & $0,021 \pm 0,007$ \\
\hline & $30 / 03$ & $<0,013$ & & $19 / 05$ & $0,025 \pm 0,007$ \\
\hline & $01 / 04$ & $0,008 \pm 0,005$ & & $23 / 09$ & $<0,017$ \\
\hline & $04 / 04$ & $0,010 \pm 0,003$ & & & \\
\hline & $06 / 04$ & $<0,011$ & & & \\
\hline & 08/04 & $0,009 \pm 0,004$ & & & \\
\hline & $11 / 04$ & $0,011 \pm 0,006$ & & & \\
\hline & $13 / 04$ & $<0,013$ & & & \\
\hline & $18 / 04$ & $<0,012$ & & & \\
\hline & $27 / 04$ & $<0,007$ & & & \\
\hline & $01 / 06$ & $<0,011$ & & & \\
\hline & $07 / 07$ & $<0,006$ & & & \\
\hline & $26 / 07$ & $<0,011$ & & & \\
\hline \multirow{6}{*}{$\begin{array}{r}\text { Viande de } \\
\text { brebis }\end{array}$} & $18 / 04$ & $0,020 \pm 0,006$ & & & \\
\hline & $16 / 05$ & $0,037 \pm 0,010$ & & & \\
\hline & $27 / 06$ & $0,007 \pm 0,003$ & & & \\
\hline & $26 / 07$ & $<0,011$ & & & \\
\hline & $12 / 09$ & $<0,010$ & & & \\
\hline & $07 / 11$ & $<0,026$ & & & \\
\hline
\end{tabular}




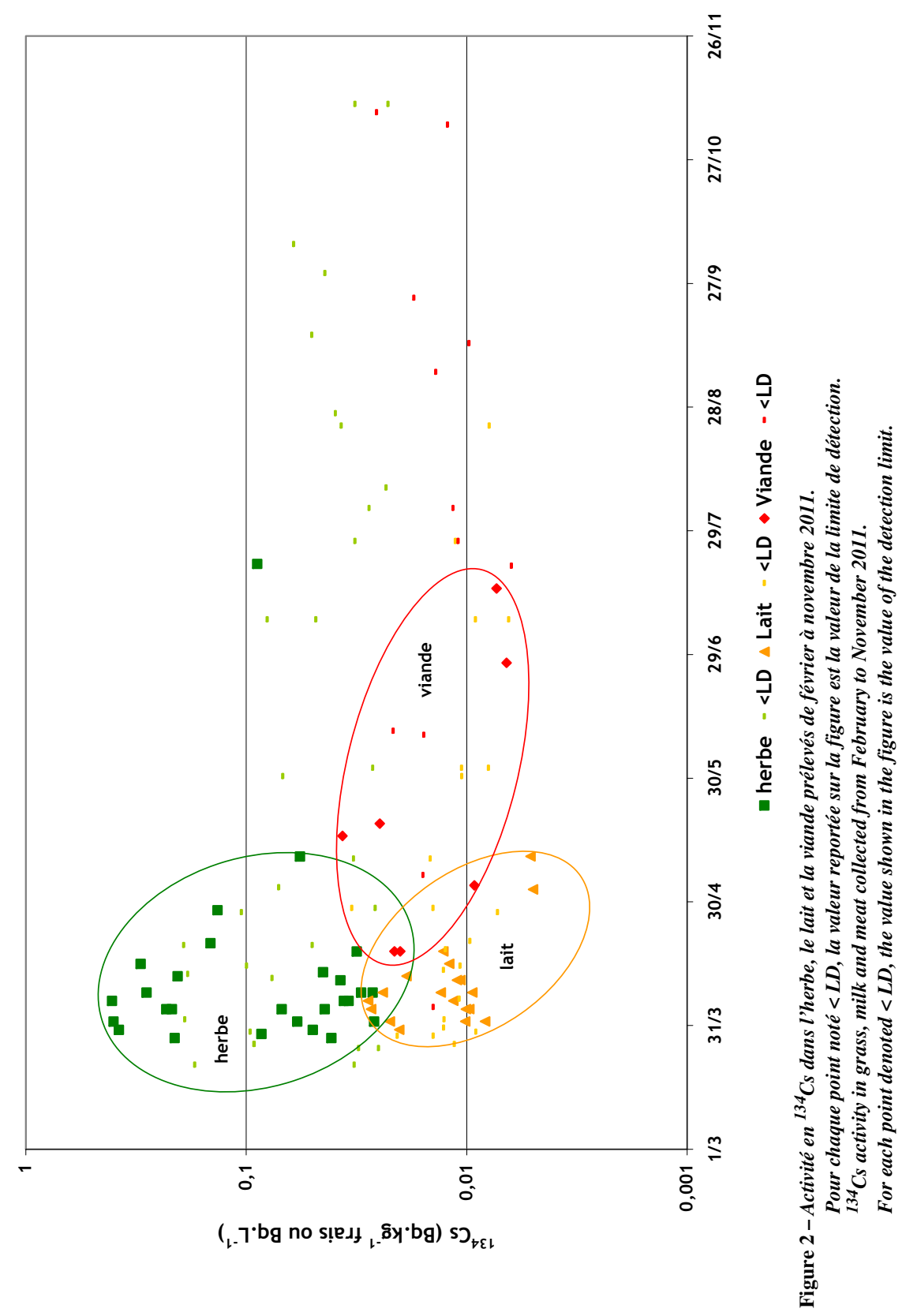




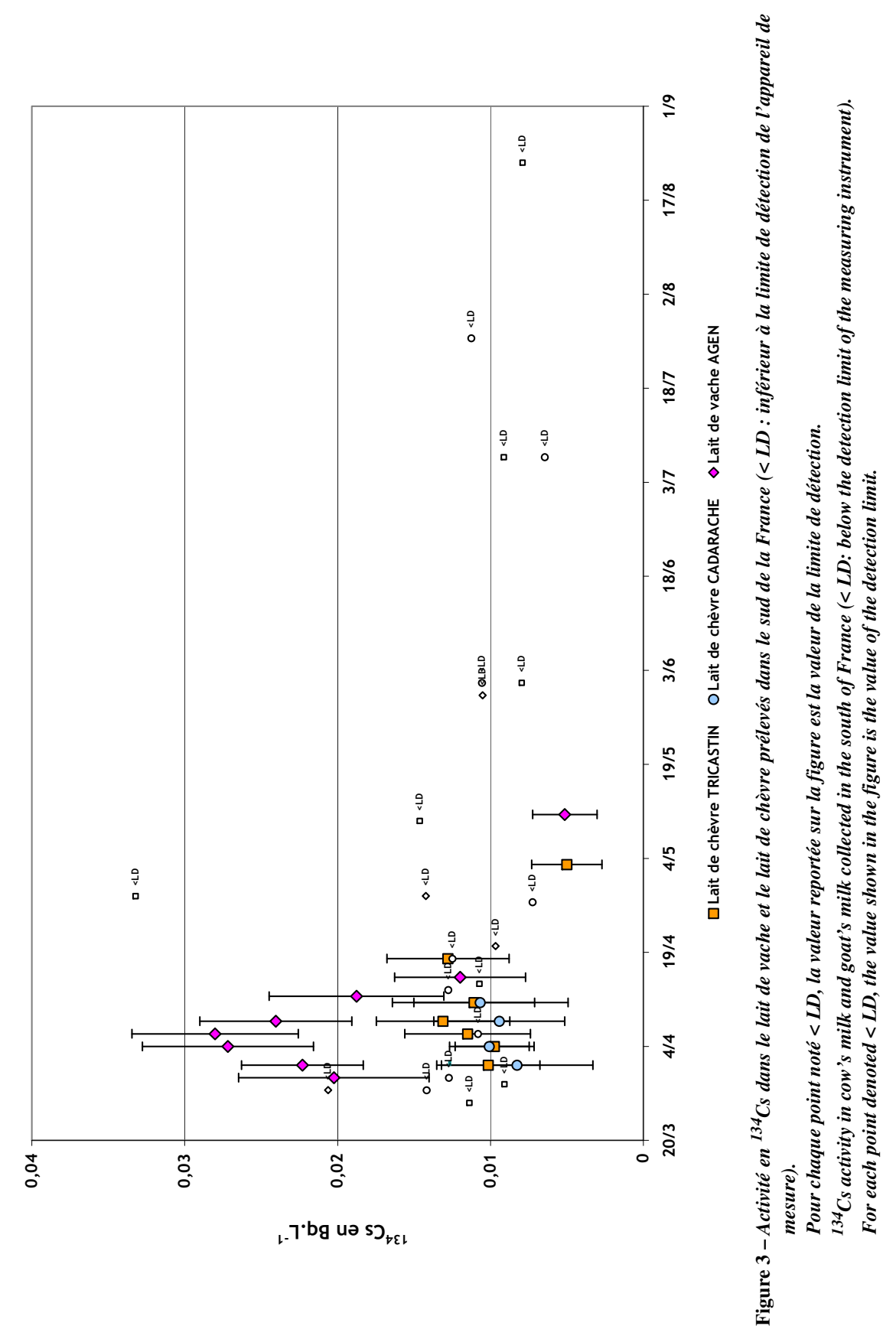




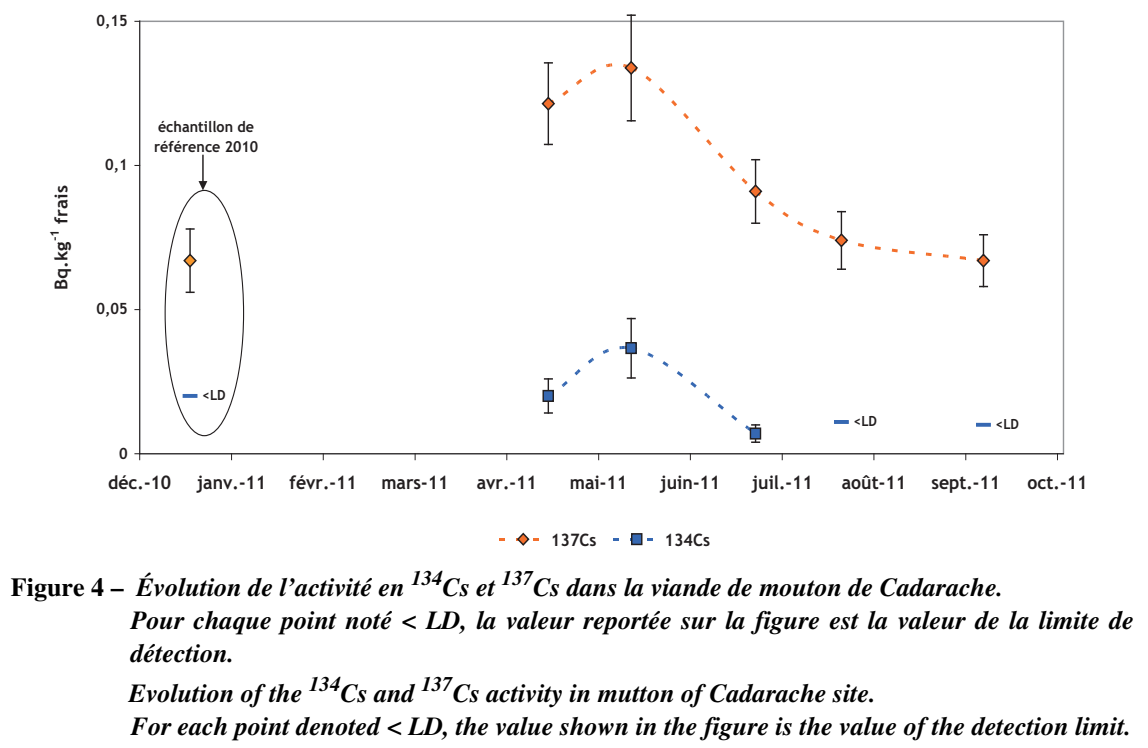

(Fig. 4). Les activités les plus fortes sont observées à la mi-mai $\left(0,037\right.$ Bq.kg $\left.{ }^{-1}\right)$. Puis, l'activité diminue (0,007 Bq. $\mathrm{kg}^{-1}$ fin juin) pour passer ensuite sous la limite de détection. De plus, l'activité en ${ }^{137} \mathrm{Cs}$ présente des variations temporelles similaires à celles du ${ }^{134} \mathrm{Cs}$. Sur cette station, l'activité en césium-137 ajoutée par les dépôts provenant de Fukushima-Daichi est bien visible, surtout lorsque les activités mesurées au printemps 2011 (activité maximale : $0,13 \mathrm{~Bq} \cdot \mathrm{kg}^{-1}$ ) sont comparées à une mesure réalisée l'année précédente dans les Alpes-de-HauteProvence $\left(0,06 \mathrm{~Bq} \cdot \mathrm{kg}^{-1}\right)$. En ce point, le rapport isotopique ${ }^{134} \mathrm{Cs} /{ }^{137} \mathrm{Cs}$ de 0,1 à 0,3 reste inférieur à celui qui a été mesuré dans l'air (environ 1, selon Masson et al., 2011). Ce qui confirme qu'une partie du ${ }^{137} \mathrm{Cs}$ mesuré dans la viande provient des retombées anciennes (Tchernobyl et essais atmosphériques).

En dépit du faible nombre de mesures d'activité en ${ }^{134}$ Cs supérieures aux limites de détections, des différences notables peuvent être observées au sein d'une même espèce animale et d'une espèce à l'autre (Fig. 5). Les activités les plus fortes sont mesurées dans la viande de mouton $\left(0,02\right.$ à $\left.0,037 \mathrm{~Bq} \cdot \mathrm{kg}^{-1}\right)$ et de veau $(0,021$ à $0,025 \mathrm{~Bq} \cdot \mathrm{kg}^{-1}$ ) prélevées en avril-mai. La seule mesure d'activité significative sur les cinq échantillons de viande de taureau prélevés dans le même intervalle de temps, témoigne d'une concentration plus faible $\left(0,009 \mathrm{~Bq} \cdot \mathrm{kg}^{-1}\right)$. Un niveau d'activité comparable est observé dans la viande de bœuf prélevée au cours du mois de juillet $\left(0,007 \mathrm{~Bq} \cdot \mathrm{kg}^{-1}\right)$. 


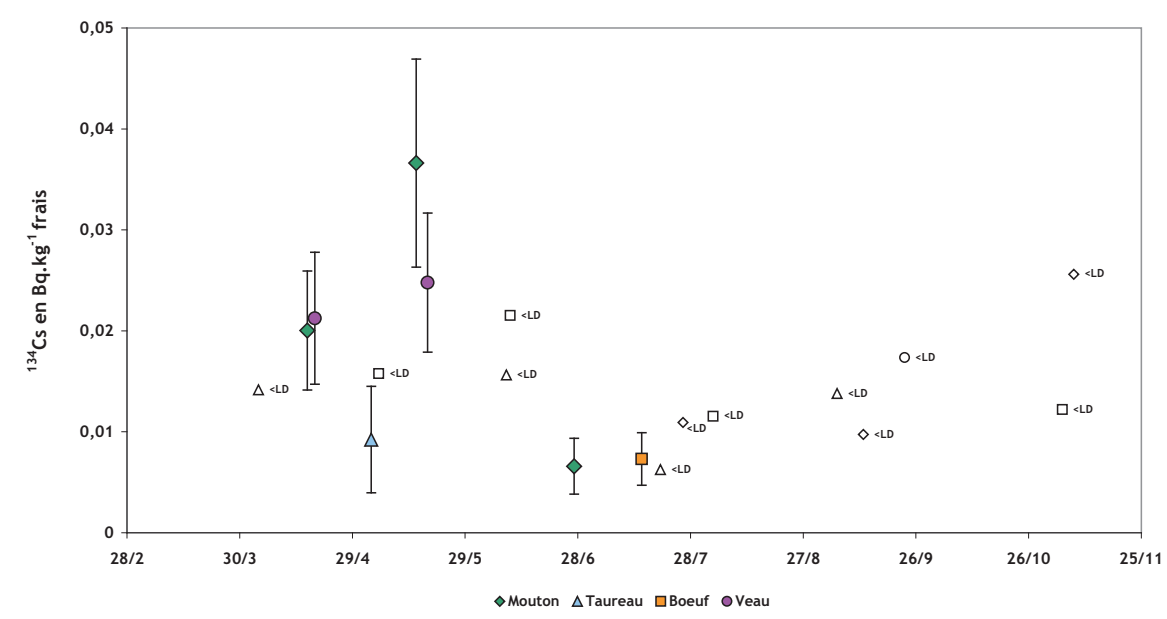

Figure 5 - Activité en ${ }^{134}$ Cs dans les échantillons de viande du sud de la France.

Pour chaque point noté < LD, la valeur reportée sur la figure est la valeur de la limite de détection.

${ }^{134}$ Cs activity in meat samples in the south of France.

For each point denoted $<L D$, the value shown in the figure is the value of the detection limit.

\section{Interprétations}

\subsection{Variabilité spatio-temporelle des activités}

L'activité observée dans la végétation de prairie $(0,026 \pm 0,009$ à $0,41 \pm$ $\left.0,15 \mathrm{~Bq} \cdot \mathrm{kg}^{-1}\right)$ est comparable à celle mesurée en Allemagne $(0,06 \pm 0,01$ à $0,32 \pm$ 0,03 Bq. $\mathrm{kg}^{-1}$ ) (Pittauerová et al., 2011) (Tab. II). L'activité de ce radioisotope dans le lait de vache $\left(0,005 \pm 0002\right.$ à $0,028 \pm 0,005$ Bq. $\left.\cdot \mathrm{L}^{-1}\right)$ et le lait de chèvre $\left(0,005 \pm 0,002\right.$ à $\left.0,013 \pm 0,004 \mathrm{~Bq} . \mathrm{L}^{-1}\right)$ est plus faible que celle qui est observée dans ces matrices $\left(0,041\right.$ à 0,067 et 0,059 à $0,069 \mathrm{~Bq} \cdot \mathrm{L}^{-1}$, respectivement $)$ en Italie, dans la région de Milan (Ioannidou et al., 2012).

L'activité en ${ }^{134} \mathrm{Cs}$ dans la végétation de prairie prélevée dans la région d'Agen $\left(0,06 \pm 0,03\right.$ à $\left.0,41 \pm 0,15 \mathrm{~Bq} \cdot \mathrm{kg}^{-1}\right)$ est comparable aux résultats des mesures provenant de la région de Bordeaux $\left(0,22\right.$ à $\left.0,53 \mathrm{~Bq} \cdot \mathrm{kg}^{-1}\right)$ (Perrot et al., 2012) (Tab. II). En comparaison, les activités sont plus faibles dans le sud-est du pays $\left(0,026 \pm 0,009\right.$ à $\left.0,22 \pm 0,07 \mathrm{~Bq} \cdot \mathrm{kg}^{-1}\right)$. Le lait de vache échantillonné dans le sud-ouest de la France témoigne également d'une activité légèrement supérieure à celles mesurées dans les échantillons de lait de chèvre des sites du sud-est. Les mesures de l'activité en ${ }^{131}$ I effectuées également au printemps 2011 et sur ces 
TABLEAU II

Activité en ${ }^{134} \mathrm{Cs}$ dans les échantillons d'herbe, de lait et de viande prélevés en France et en Europe en 2011.

${ }^{134} \mathrm{Cs}$ activity measured in samples of grass, milk and meat collected in France and Europe in 2011.

\begin{tabular}{|c|c|c|c|c|c|}
\hline & 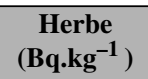 & $\begin{array}{l}\text { Lait de chèvre } \\
\left(\mathrm{Bq} . \mathrm{L}^{-1}\right)\end{array}$ & $\begin{array}{l}\text { Lait de vache } \\
\left(\mathrm{Bq} \cdot \mathrm{L}^{-1}\right)\end{array}$ & $\begin{array}{c}\text { Viande } \\
\left({\left.\mathrm{Bq} . \mathrm{kg}^{-1}\right)}^{-1}\right.\end{array}$ & \\
\hline France & $\begin{array}{c}0,02 \pm 0,006 \\
\text { à } \\
0,40 \pm 0,07\end{array}$ & $\begin{array}{c}0,008 \pm 0,005 \\
\text { à } \\
0,013 \pm 0,004\end{array}$ & $\begin{array}{c}0,005 \pm 0,002 \\
\text { à } \\
0,028 \pm 0,005\end{array}$ & $\begin{array}{c}0,007 \pm 0,003 \\
\quad \text { à } \\
0,037 \pm 0,010\end{array}$ & présente étude \\
\hline France & $\begin{array}{c}0,2 \pm 0,1 \\
\text { à } \\
1,6 \pm 0,4\end{array}$ & - & - & - & Evrard et al., 2012 \\
\hline France & $\begin{array}{c}0,22 \pm 0,05 \\
\text { à } \\
0,63 \pm 0,11\end{array}$ & - & - & - & Perrot et al., 2012 \\
\hline Allemagne & $\begin{array}{c}0,062 \pm 0,014 \\
\text { à } \\
0,32 \pm 0,03\end{array}$ & - & - & - & $\begin{array}{c}\text { Pittauerová et al., } \\
2011\end{array}$ \\
\hline Italie & $0,021 \pm 0,012$ & $\begin{array}{c}0,059 \pm 0,044 \\
\text { à } \\
0,069 \pm 0,026\end{array}$ & $\begin{array}{c}0,041 \pm 0,024 \\
\text { à } \\
0,067 \pm 0,035\end{array}$ & - & $\begin{array}{l}\text { Ioannidou et al., } \\
2012\end{array}$ \\
\hline
\end{tabular}

mêmes sites de prélèvement témoignent de la même tendance spatiale, avec des activités légèrement accentuées dans le sud-ouest de la France (Parache et al., 2011).

De plus, les variations des activités dans la végétation et le lait sont couplées au cours de la période d'étude, suggérant un transfert du césium dans un bref délai entre ces deux compartiments. Dans le lait et la végétation, la phase de dépôt de la radioactivité est à l'origine de l'augmentation de l'activité observée (Parache et al., 2011). Puis, dans un deuxième temps, l'arrêt du dépôt et la croissance des végétaux (particulièrement marquée au printemps) sont la cause de la diminution rapide de l'activité en radiocésium des végétaux et du lait. Ainsi, les variations similaires des activités en ${ }^{134} \mathrm{Cs}$ observées dans la végétation des prairies et dans le lait des troupeaux confirment que le lait constitue un indicateur robuste de l'intensité du dépôt atmosphérique, de sa variabilité spatiale et du transfert au cours du temps.

En dehors de la présente étude, aucune mesure de l'activité en ${ }^{134} \mathrm{Cs}$ effectuée en 2011 dans la viande prélevée en Europe n'est disponible à ce jour dans la littérature (Tab. II). Malgré le faible nombre de mesures de l'activité dans la viande, on peut observer que ce compartiment témoigne d'un retard de quelques jours en comparaison de la végétation et du lait. Ainsi, l'activité en ${ }^{134} \mathrm{Cs}$ dans la viande apparaît avec un retard de quinze jours environ par rapport au lait. De plus, l'activité maximale dans la viande semble atteinte vers le 15 mai, soit avec un 
décalage d'environ quarante jours par rapport aux valeurs maximales atteintes dans la végétation. Ceci s'explique par le fait que l'accumulation du césium dans la viande est un processus relativement lent (Daburon, 2000).

\section{2. Évaluation des coefficients de transfert}

À partir des mesures réalisées dans la viande et le lait des animaux, les coefficients de transfert ont été évalués en tenant compte de la quantité de végétaux frais ingérés ( 3 et $50 \mathrm{~kg} \cdot \mathrm{j}^{-1}$ pour la chèvre et les bovins, respectivement) et de l'activité de l'herbe mesurée dans l'étude, à l'aide de l'équation (1) :

Coefficient de transfert au lait $(\mathrm{Fm})$ ou à la viande $(\mathrm{Ff})$ :

$$
\frac{\text { Concentration en } \left.{ }^{134} \mathrm{Cs} \text { du lait (en Bq.L }{ }^{-1}\right) \text { ou de la viande }\left(\mathrm{Bq}_{\mathrm{kgg}}{ }^{-1}\right)}{\text { Activité en }{ }^{134} \mathrm{Cs} \text { ingérée quotidiennement }\left(\mathrm{Bq} \cdot \mathrm{j}^{-1}\right)} \text {. }
$$

Les incertitudes sur les facteurs de transfert ont été calculées à partir des incertitudes analytiques de l'activité en ${ }^{134} \mathrm{Cs}$ dans les matrices, sous l'hypothèse de distribution gaussienne des erreurs.

Le coefficient de transfert est exprimé en $\mathrm{j} . \mathrm{L}^{-1}$ pour le lait $(\mathrm{Fm})$ et en $\mathrm{j} . \mathrm{kg}^{-1}$ pour la viande $(\mathrm{Ff})$ et doit théoriquement être calculé à l'équilibre lors d'une contamination continue (Daburon, 2000). Or, en raison de l'évolution de l'activité de l'herbe, cet équilibre n'a pas été atteint. Dans le cas du lait, l'activité ingérée a été calculée avec l'activité de l'herbe mesurée le jour précédent la mesure du lait. Cette simplification de la période d'intégration est rendue acceptable par le transfert rapide du césium vers les glandes mammaires dont rendent compte les courtes périodes d'élimination effective fournies dans la littérature (de l'ordre de 1 à 2 jours) (Müller et Pröhl, 1993). Dans le cas de la viande, la quantité incorporée a été calculée sur la base de l'activité moyenne de l'herbe mesurée au cours des quinze jours précédents. Ce délai d'intégration des incorporations successives tient compte d'une accumulation progressive du césium dans le muscle. Ce délai de quinze jours est court au regard des périodes d'élimination de cinquante jours fournies dans la littérature. Cependant, les différents calculs montrent que le choix de ces périodes/délais influe de manière peu significative sur la valeur du $\mathrm{Ft}^{1}$.

La variabilité des activités dans les échantillons de viande des différents animaux est proche de la variabilité des facteurs de transfert proposés par la

\footnotetext{
1 Valeurs du facteur de transfert au lait Fm $\left(\mathrm{j} . \mathrm{L}^{-1}\right)$ ou à la viande $\mathrm{Ff}\left(\mathrm{j} \cdot \mathrm{kg}^{-1}\right)$ selon le laps de temps considéré pour le calcul de l'incorporation :

- Fm chèvre : 0,093 (délai 2 jours) / 0,089 (délai 8 jours),

- Fm vache : 0,0014 (délai 2 jours) / 0,0016 (délai 8 jours),

- Ff mouton : 0,22 (délai 2 jours) / 0,22 (délai 10 jours) / 0,23 (délai 30 jours).
} 
littérature. Ainsi, les viandes qui témoignent des activités les plus élevées (mouton et veau) sont celles qui présentent les coefficients de transfert les plus importants, en moyenne $2,7 \times 10^{-1}$ (IAEA, 2010) et 0,35 à $0,6 \mathrm{j} \cdot \mathrm{kg}^{-1}$ (Voigt et al., 1989 ; Ajlouni et al., 2008), respectivement. À l'opposé, la viande de bovin présente un coefficient de transfert moyen un peu plus faible $\left(2,2 \times 10^{-2}\right.$ j.kg $\left.{ }^{-1}\right)$ qui expliquerait les valeurs globalement plus modestes en comparaison des autres espèces (IAEA, 2010).

En ce qui concerne la viande, le seul coefficient de transfert qui ait été évalué, en l'occurrence celui de la viande de mouton prélevée à proximité du site de Cadarache pour lequel on dispose d'une série de données relativement complète (Ff : $2,2 \times 10^{-1} \pm 0,9 \times 10^{-1} \mathrm{j} . \mathrm{kg}^{-1}$ ), est proche de la valeur moyenne citée précédemment. Les coefficients de transfert moyens, calculés pour cette étude, au lait de chèvre (sites du Tricastin et de Cadarache) et de vache sont de $9,01 \times 10^{-2} \pm$ $4,8 \times 10^{-2}$ et $1,4 \times 10^{-3} \pm 0,5 \times 10^{-3} \mathrm{j} . \mathrm{L}^{-1}$, respectivement. Ces résultats sont également proches des valeurs moyennes de $1,3 \times 10^{-1} \mathrm{j} . \mathrm{L}^{-1}$ pour le lait de chèvre et de $6,1 \times 10^{-3} \mathrm{j} . \mathrm{L}^{-1}$ pour le lait de vache, disponibles dans la littérature (Howard et al., 2009).

\section{Conclusion}

Les prélèvements et les mesures de l'activité en ${ }^{134} \mathrm{Cs}$ réalisés au cours du printemps et de l'été 2011 par l'IRSN ont permis d'évaluer, dans les conditions réelles des dépôts provenant des rejets de Fukushima-Daichi, la chronologie des transferts du césium dans les compartiments du milieu terrestre et notamment l'accumulation de ce radionucléide au fil du temps dans la viande des animaux. Des coefficients de transfert à la viande de mouton, aux laits de vache et de chèvre représentatifs des sites étudiés sont également proposés.

En termes d'impact sanitaire, les résultats acquis par l'IRSN (IRSN, 2011) ont montré que les doses potentiellement reçues en France après l'accident de Fukushima-Daichi sont très faibles et qu'à aucun moment, les concentrations en radionucléides d'origine artificielle (iode-131, césium-134 et césium-137) mesurées dans les différents compartiments de l'environnement n'ont présenté un risque environnemental ou sanitaire.

\section{RÉFÉRENCES}

AFNOR (2010) NF ISO 11929 Détermination des limites caractéristiques (seuil de décision, limite de détection et extrémités de l'intervalle de confiance) pour mesurages de rayonnements ionisants, Principes fondamentaux et applications, mai 2010.

Ajlouni A.W., Abu-Haija O., Abdelsalam M., Al-Rabai' ah H. (2008) Nuclear fission products behavior in the environment, J. of Appl. Sci. Environm. Sanitation 3, 109-116. 
Daburon F. (2000) Chaîne Alimentaire, dans Le cesium de l'environnement à l'homme (D. Robeau, F. Daburon, H. Métivier, Eds.) pp.151-186. EDP Sciences, Les Ulis.

Evrard O., Van Beek P., Gateuille D., Pont V., Lefèvre I., Lansard B., Bonté P. (2012) Evidence of the radioactive fallout in France due to the Fukushima nuclear accident, J. Environ. Radioact. 114, 54-60.

Howard B.J., Beresford N.A., Barnett C.L., Fesenko S. (2009) Radionuclide transfert to animal products: revised recommended transfert coefficient values, J. Environ. Radioact. 100, 263-273.

IAEA (2010) Technical reports series $\mathrm{N}^{\circ} 472$, Handbook of parameter values for the prediction of radionuclide transfer in terrestrial and freshwater environments, Vienne, pp. 82-99.

Ioannidou A., Manenti S., Gini L., Groppi F. (2012) Fukushima fallout at Milano, Italy, J. Environ. Radioact. 114, 119-125.

JCGM (2008). JCGM 100:2008. Evaluation of measurement data - Guide to the expression of uncertainty in measurement - GUM 1995 with minor corrections, septembre 2008.

IRSN (2011) Analyse de l'impact de l'accident de Fukushima en France (métropole et DROM-COM) à partir des résultats de la surveillance renforcée de la radioactivité de l'environnement, rapport IRSN/DEI 2011-01, 90 p.

Masson O., Baeza A., Bieringer J., Brudecki K., Bucci S., Cappai M., Carvalho F.P., Connan O., Cosma C., Dalheimer A., Didier D., Depuydt G., De Geer L.E., De Vismes A., Gini L., Groppi F., Gudnason K., Gurriaran R., Hainz D., Halldórsson Ó., Hammond D., Hanley O., Holeý K., Homoki Zs., Ioannidou A., Isajenko K., Jankovic M., Katzlberger C., Kettunen M., Kierepko R., Kontro R., Kwakman P.J.M., Lecomte M., Leon Vintro L., Leppänen A.-P., Lind B., Lujaniene G., McGinnity P., McMahon C., Malá H., Manenti S., Manolopoulou M., Mattila A., Mauring A., Mietelski J.W., Møller B., Nielsen S.P., Nikolic J., Overwater R.M.W., Pálsson S.E., Papastefanou C., Penev I., Pham M.K., Povinec P.P., Ramebäck H., Reis M.C., Ringer W., Rodriguez A., Rulík P., Saey P.R.J., Samsonov V., Schlosser C., Sgorbati G., Silobritiene B.V., Söderström C., Sogni R., Solier L., Sonck M., Steinhauser G., Steinkopff T., Steinmann P., Stoulos S., Sýkora I., Todorovic D., Tooloutalaie N., Tositti L., Tschiersch J., Ugron A., Vagena E., Vargas A., Wershofen H., Zhukova O. (2011) Tracking of airborne radionuclides from the damaged Fukushima Dai-Ichi nuclear reactors by European networks, Environ. Sci. Technol. 45, 7670-7677.

Müller H., Pröhl G. (1993) ECOSYS-87 A dymanic model for assessing radiological consequences of nuclear accidents, Health Phys. 64, 232-249.

Parache V., Pourcelot L., Roussel-Debet S., Orjollet D., Leblanc F., Soria C., Gurriaran R., Renaud Ph., Masson O. (2011) Transfer of ${ }^{131}$ I from Fukushima to the vegetation and milk in France, Environ. Sci. Technol. 45, 9998-1003.

Perrot F., Hubert Ph., Marquet Ch., Pravikoff M.S., Bourquin P., Chiron H., Guernion P.-Y., Nachab A. (2012) Evidence of ${ }^{131}$ I and ${ }^{134,137}$ Cs activities in Bordeaux, France due to the Fukushima nuclear accident, J. Environ. Radioact. 114, 61-65.

Pittauerová D., Hettwig B., Fischer H.W. (2011) Fukushima fallout in Northwest German environmental media, J. Environ. Radioact. 102, 877-880.

Voigt G., Pröhl G., Müller H., Bauer T., Lindner J.P., Probstmeier G., Röhrmoser G. (1989) Determination of the transfer of cesium and iodine from feed into domestic animals, Sci. Total Environ. 85, 329-338. 